\title{
Olfactory Bulb Recovery after Early Sensory Deprivation
}

\author{
D. M. Cummings, H. E. Henning, and P. C. Brunjes \\ Neuroscience Program and Department of Psychology, University of Virginia, Charlottesville, Virginia 22903
}

Olfactory bulbs retain the ability to acquire new neurons throughout life. Unilateral olfactory deprivation during the first postnatal month in rats results in a dramatic reduction in the size of the experimental olfactory bulb. Part of this reduction is attributable to the death of neurons and glia. To examine the regenerative capacity of the juvenile olfactory bulb, we developed a technique for reversible olfactory deprivation. Reversible blockade from postnatal day 1 (P1) to P20 or P30 results in reduced bulb volume and tyrosine hydroxylase immunostaining, and decreased depth in the olfactory mucosa. In another experiment, normal stimulation was restored for varying periods of time, and experimental and control bulb volumes were measured. Recovery of bulb size occurs after $40 \mathrm{~d}$ of normal stimulation. Rats injected with a thymidine analog to label dividing cells during the recovery period revealed that rescue results at least in part from the addition of new neurons and glia. Thus, cells born after the return of normal levels of environmental stimulation can replace some of the neurons and glia that are lost during olfactory deprivation. This system can be used to study mechanisms that underlie neuronal regeneration in the maturing mammalian brain.

Key words: neuronal regeneration; sensory deprivation; unilateral naris closure; rostral migratory stream; bromodeoxyuridine (BrdU); olfactory bulb; development
An ongoing focus in neurobiology concerns the limited ability of the mammalian CNS to recover after neuronal cell death or injury. Although transplantation of fetal brain tissue into the adult nervous system has led to promising results (Widner, 1993), endogenous sources of replacement neurons have been presumed to be nonexistent after the early neonatal period. The olfactory system offers an exception to this rule, however, because stem cells differentiate into neurons in both the sensory epithelium and first order relay, the olfactory bulb, well into adulthood (Altman, 1963; Bayer, 1983; Kaplan et al., 1985; Farbman, 1992). In fact, a great deal of research has recently focused on the proliferative region located ventral to the anterior lateral ventricles, the subventricular zone (SVZ). Cells from the SVZ migrate along the "rostral migratory stream" (Altman, 1969) and into the olfactory bulbs where many differentiate into interneurons (FrazierCierpial and Brunjes, 1989; Luskin, 1993; Lois and AlvarezBuylla, 1994). The purpose for this lifelong addition of new neurons is unknown, although it is possible that cell incorporation is regulated by the availability of synaptic space and/or physiological activity.

Afferent activity does play a significant role in bulb maturation. For example, when half of the olfactory system is deprived of normal stimulation during early life (by reducing airflow through one side of the nasal cavity), there is a dramatic (25\%) decrease in the size of the ipsilateral bulb (Meisami, 1976; Brunjes, 1994). A substantial literature has evolved examining the changes oc curring after neonatal naris closure (Brunjes, 1994). Nearly all bulb layers are reduced in size 2 weeks after occlusion. These

Received Jan. 24, 1997; revised July 3, 1997; accepted July 10, 1997.

This study was supported by National Institutes of Health (DC00338 and HD07323). We thank Dr. Tammy Dellovade for technical advice and comments on this manuscript and Mr. Brian Knab for technical assistance.

Correspondence should be addressed to Dr. Peter C. Brunjes, 102 Gilmer Hall, University of Virginia, Charlottesville, VA 22903.

Dr. Cummings' present address: Department of Anatomy and Neurobiology, University of Maryland at Baltimore, School of Medicine, 685 W. Baltimore Street, Baltimore, MD 21201.

Copyright (C) 1997 Society for Neuroscience $0270-6474 / 97 / 177433-08 \$ 05.00 / 0$ changes result from increased cell death and not from either decreased cellular proliferation or rates of migration (Brunjes, 1994; Najbauer and Leon, 1995).

Until now, unilateral olfactory deprivation has been accomplished by permanent surgical closure of an external naris. We have developed a method for reversibly blocking half of the nasal cavity to investigate whether "deprived" bulbs possesses the ability to recover their normal size. This method, based on the work of Kucharski and Hall (1987), involves the insertion of removable nose plugs. As demonstrated below, the technique results in changes similar to those seen after permanent naris closure. Because the rostral migratory stream provides cells throughout life in rodents, we predicted that recovery might be possible once stimulation is returned. The possibility was tested by blocking rats' nares for a period of time long enough to produce a decrease in bulb volume and then labeling cells born during the recovery period with the thymidine analog bromodeoxyuridine (BrdU) (Gratzner, 1982). Indeed, we found both that bulb volume recovers and that substantially more BrdU-labeled cells are found after the return of normal olfactory stimulation. Immunocytochemistry for a specific neural marker revealed that many of the new cells are interneurons, and counts revealed that more neurons are added to the previously deprived bulb. Thus, we present a mammalian model system for examining neuronal regeneration in the maturing CNS.

\section{MATERIALS AND METHODS}

All experimental procedures met National Institutes of Health guidelines and were endorsed by the Animal Use and Care Committee of the University of Virginia. Animals used in this study were offspring of Long-Evans hooded rats (Charles Rivers Breeding Laboratories, Wilmington, MA). On postnatal day 1 (P1) litters were culled to 10-12 pups, and reversible naris closure was begun by inserting removable nose plugs into the right external nares.

\section{Reversible naris closure}

The method of nose plug construction was modified from a design used in Dr. W. G. Hall's laboratory (Kucharski and Hall, 1987). Nose plugs were constructed out of polyethylene (PE) tubing (Becton Dickinson, 


\section{CONSTRUCTION OF NOSE PLUGS}

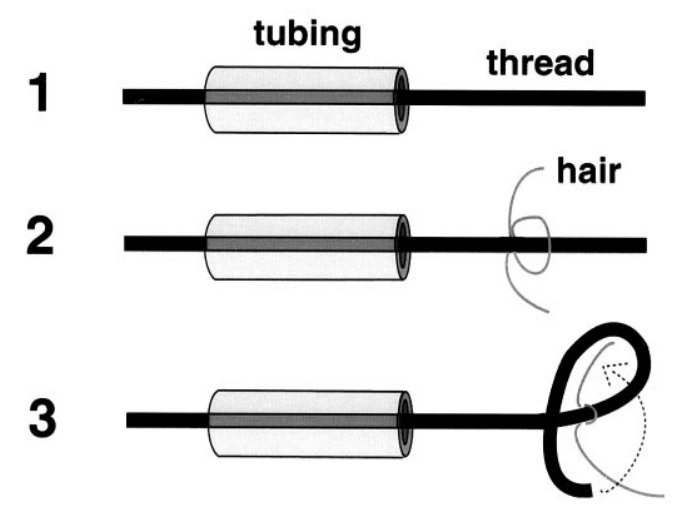

4

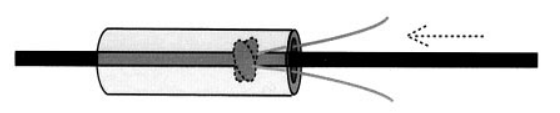

5

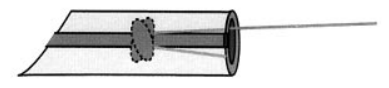

Figure 1. Diagram indicating how plugs were constructed. (1) A length of suture was threaded through a piece of tubing. (2) A piece of hair (or strands of dental floss) was tied around the thread, and (3) the thread was tied into a knot around the hair. (4) The knot was pulled into the lumen of the tubing, and (5) the ends of thread and hair were trimmed until only $2 \mathrm{~mm}$ of hair remained extending from the tubing. The opposite end of tubing was beveled for ease of insertion.

\begin{tabular}{llll}
\hline \multicolumn{4}{l}{ Table 1. Materials used in the construction of different sized nose plugs } \\
Tubing size & Outer diameter $(\mathrm{mm})$ & Thread size & Hair/floss \\
\hline PE10 & 0.61 & $5-0$ & Hair \\
PE50 & 0.96 & $3-0$ & Hair \\
PE20 & 1.09 & $3-0$ & Floss \\
PE60 & 1.22 & 0 & Floss \\
PE90 & 1.27 & 2 & Floss \\
PE100 & 1.52 & 2 & Floss \\
\hline
\end{tabular}

Parsippany, NJ), silk surgical suture thread, and either human hair or filaments obtained from unwaxed dental floss (Fig. 1). When the plugs were inserted properly, only $2 \mathrm{~mm}$ of hair or floss extended from the external naris. Thus, although animals were unable to grasp the plugs, they could be removed with forceps at any time.

We used varying sizes of tubing (PE10-PE100) and silk surgical suture thread (sizes 5-0 to 2) to construct plugs of increasing size (Table 1). As pups grew, plugs were replaced with larger ones every 3-6 d. Before plug insertion or replacement, young pups (P1-P5) were anesthetized with hypothermia, and older animals were anesthetized with Metofane (Pitman-Moore, Mundelein, IL). Once each animal was lightly anesthetized, the existing plug was removed by pulling on the hair with forceps, and a plug of slightly larger length and diameter was coated with a small amount of petroleum jelly and inserted. Plug replacement was routinely accomplished in $<1 \mathrm{~min}$ to minimize exposure to normal olfactory stimulation. After plugs were replaced, pups were examined to ensure that normal respiration had commenced before they were returned to the dam.

\section{Laminar volume measurements}

In the first study, pups were reversibly occluded from P1 to P20 or P1 to P30 to assess the reliability of the technique as compared with permanent naris closure using cautery (Brunjes, 1994). In subsequent groups, pups were reversibly occluded from P1 to P20 and then allowed to "recover" for 10,20 , or $40 \mathrm{~d}$ with access to normal olfactory stimulation. On the appropriate day, each animal was overdosed with barbiturate anesthetics and perfused transcardially with a fixative containing $10 \%$ glycerol and $0.5 \%$ formalin. Brains were removed, frozen rapidly in isopentane at $-70^{\circ} \mathrm{C}$, and stored at this temperature until sectioning. Olfactory bulbs were sectioned $(30 \mu \mathrm{m})$, post-fixed, stained with cresyl violet, dehydrated, cleared, and coverslipped using D.P.X. mounting medium (Aldrich, Milwaukee, WI). Volumes of the glomerular (GLM), external plexiform (EPL), and combined mitral, internal plexiform, and granule cell (GCL) layers in the right and left olfactory bulbs were measured. Because of the clearly laminated structure of the bulb, layers are easily delineated, with great interobserver reliability in their measurement. We used a standardized technique used routinely by this and other laboratories (Brunjes and Borror, 1983). Images of sections were projected at $40 \times$ onto a digitizing tablet, and the area of each lamina was measured in every tenth section using Sigma Scan computer software (Jandel Scientific, Corte Madera, CA). Each lamina was measured twice, and means were calculated to increase the accuracy of the measurement. Then, the volume of each layer in the right (experimental) and left (control) olfactory bulbs was calculated as described previously. The percentage difference in laminar volume was computed using the formula $((L-R) / 1 \times 100)$, where $L$ and $R$ are the volumes of the left and right bulbs, respectively.

\section{Tyrosine hydroxylase (TH) immunocytochemistry and quantification}

Another group of animals was reversibly occluded from P1 to P30, and olfactory bulb sections were processed for $\mathrm{TH}$ immunocytochemistry $(n=5)$. On P30, rats were overdosed with barbiturate anesthetics and perfused transcardially with $0.1 \mathrm{~m}$ PBS followed by Bouin's fixative. The brains were then dehydrated in graded alcohols, embedded in paraffin, and sectioned coronally at $8 \mu \mathrm{m}$. Three sections, separated by at least 400 $\mu \mathrm{m}$, were mounted onto gelatin-subbed slides, incubated on a $37^{\circ} \mathrm{C}$ hot plate overnight, deparaffinized in xylenes, and rehydrated through graded alcohols. Slides were rinsed in $0.1 \mathrm{M}$ PBS and incubated in 3\% $\mathrm{H}_{2} \mathrm{O}_{2}$ to block endogenous peroxidases. Sections were then processed using ABC-immunocytochemistry with anti-TH primary antiserum (Incstar, Stillwater, MN) (dilution, 1:5000) as described previously (Brunjes et al., 1992). The density of TH immunoreactivity (TH-ir) along the medial aspects of the GLM layer of experimental and control olfactory bulbs was measured (as the percentage of test area covered by immunoreactive areas) using the grain-counting package of the MCID M4 imaging software (Imaging Research, St. Catherines, Ontario, Canada).

\section{Olfactory mucosa}

The caudal nasal cavities from animals perfused for laminar volume measurements were removed and stored in $4 \%$ paraformaldehyde. They were decalcified overnight, dehydrated, embedded in paraffin, and sectioned coronally $(8-10 \mu \mathrm{m})$. Every 50th section was mounted onto gelatin-subbed slides, dried overnight at $37^{\circ} \mathrm{C}$, deparaffinized, and stained with hematoxylin and eosin (Lerner Laboratories, Pittsburgh, PA). On both experimental and control sides, measurements were made of epithelial thickness at two points along the nasal septum using the MCID software. These measurements were averaged, and the percentage difference between experimental and control sides of the nasal cavity was computed.

\section{$\operatorname{BrdU}$}

BrdU injections. Two groups of pups were fitted with nose plugs for P1 to P20. The first group (Group 1; $n=4$ experimental, 4 control) was then injected two times (with $8 \mathrm{hr}$ between injections) with $\operatorname{BrdU}(50 \mathrm{mg} / \mathrm{kg}$, i.p.; Sigma, St. Louis, MO) on the day after the plug was removed. The second group (Group 2; $n=3$ experimental, 3 control) received BrdU injections $30 \mathrm{~d}$ after plug removal (on P50). In this group, two BrdU injections (separated by $8 \mathrm{hr}$ ) were given daily for $3 \mathrm{~d}$ to maximize the opportunity for uptake by proliferating cells. All animals were killed with 
barbiturate anesthetics $30 \mathrm{~d}$ after the last BrdU injection, and their brains were processed for BrdU immunocytochemistry.

BrdU immunocytochemistry. All animals were perfused transcardially with 0.1 M PBS followed by Bouin's fixative. Brains were removed, embedded in paraffin, and sectioned coronally at $8 \mu \mathrm{m}$, and every 50th section was mounted onto OptiPlus positively charged slides (BioGenex, San Ramon, CA). Sections were baked for $2 \mathrm{hr}$ in a $60^{\circ} \mathrm{C}$ oven, deparaffinized in toluene, and rehydrated in graded alcohols. Afterward, slides were immersed in 20\% trypsin (Zymed Laboratories, San Francisco, $\mathrm{CA}$ ) for $10 \mathrm{~min}$ at $37^{\circ} \mathrm{C}$, rinsed in $0.1 \mathrm{M} \mathrm{PBS}$, and incubated in $2 \mathrm{~N} \mathrm{HCl}$ at $37^{\circ} \mathrm{C}$ to denature the DNA. Sections were then rinsed in borate buffer, $\mathrm{pH} 8.3$, followed by $0.1 \mathrm{M}$ PBS. Slides were transferred to $2 \%$ normal goat serum with $2 \%$ bovine serum albumin for $1 \mathrm{hr}$ to block nonspecific binding and then were incubated in mouse anti-BrdU primary antiserum (Zymed) (dilution, 1:100) for $1 \mathrm{hr}$ at room temperature. Sections were then processed through biotinylated anti-mouse secondary antisera (Dako Corporation, Carpinteria, CA) (dilution, 1:100), avidin-biotinhorseradish peroxidase complex (ABC Elite Kit; Vector Laboratories, Burlingame, $\mathrm{CA}$ ), and reacted in 3, 3' diaminobenzidine tetrahydrochloride (DAB; Sigma) as described elsewhere (Brunjes et al., 1992). Slides were then rinsed in $0.1 \mathrm{M} \mathrm{PB}$, counterstained with $0.03 \%$ methylene blue, dehydrated, cleared, and coverslipped in D.P.X. In control sections the specificity of BrdU labeling was verified by (1) omitting the primary antisera and (2) processing tissue from animals injected with vehicle alone $(n=2)$. No positive staining was observed under either of these conditions.

Double-labeling. Sections from rats injected with BrdU on P21 (Group 1) were processed with immunocytochemistry for BrdU followed by a neuronal marker. One set of tissue was processed for BrdU-ir using DAB as the chromogen as described above. Sections were then rinsed overnight in several changes of $0.1 \mathrm{M}$ PBS and incubated in rabbit antisera to calretinin (Chemicon, Temecula, CA) (dilution, 1:1000). The reaction product for the anti-calretinin primary antisera was visualized using the Vector SG peroxidase substrate kit (Vector Laboratories). The Vector SG peroxidase substrate, prepared according to kit instructions, produced a light-blue reaction product easily distinguishable from the brown reaction product associated with BrdU-ir.

Quantification of BrdU-positive profiles. Three sample sections (separated by $\sim 400 \mu \mathrm{m}$ ) were chosen from the middle of the anteriorposterior extent of the bulb. Slides were coded so that the experimenter was blind during BrdU-ir profile quantification. Using a $40 \times$ objective, BrdU-containing profiles were counted in a $1000 \mu \mathrm{m}^{2}$ strip of the GLM layer and in a $500 \mu \mathrm{m}^{2}$ strip of the GCL layer in both medial and lateral aspects of the sections.

Quantification of double-labeled profiles. In sections from recovered and control bulbs, dual-labeled cells were counted along the entire GLM $\left(\sim 5000 \mu \mathrm{m}^{2}\right.$ per section) in three sections separated by at least $400 \mu \mathrm{m}$. Tissue was coded so that the experimenter was blind to the side (e.g., recovered or control). Cells were identified as double-labeled only if they contained a nucleus that exhibited a dark-brown reaction product (BrdUir) and cytoplasm and processes that exhibited a blue reaction product (calretinin-ir). Numbers of cells per $250 \mu \mathrm{m}$ length of the GLM were calculated, and percentage differences between recovered and control bulbs were subjected to a $t$ test.

\section{RESULTS}

\section{Reversible versus permanent naris occlusion}

Laminar volume measurements

Several experiments demonstrated that our technique for reversible unilateral naris closure resulted in changes in bulb volume and cell survival similar to those found after permanent naris occlusion. First, as determined by our laminar volume measurements, animals that underwent either reversible or permanent unilateral naris occlusion from P1 to P30 exhibited comparable changes in bulb size (Fig. 2, Table 2). The mean overall reduction in experimental bulb volume of permanently occluded animals was $\sim 25 \%$ (Brunjes and Borror, 1983) (Fig. 2, filled bars), whereas the overall reduction in bulb volume of reversibly occluded rats was $\sim 20 \%$ (Fig. 2, open bars). Furthermore, in both groups the pattern of volumetric reduction was similar, with the EPL undergoing the greatest decrease. In animals reversibly occluded from
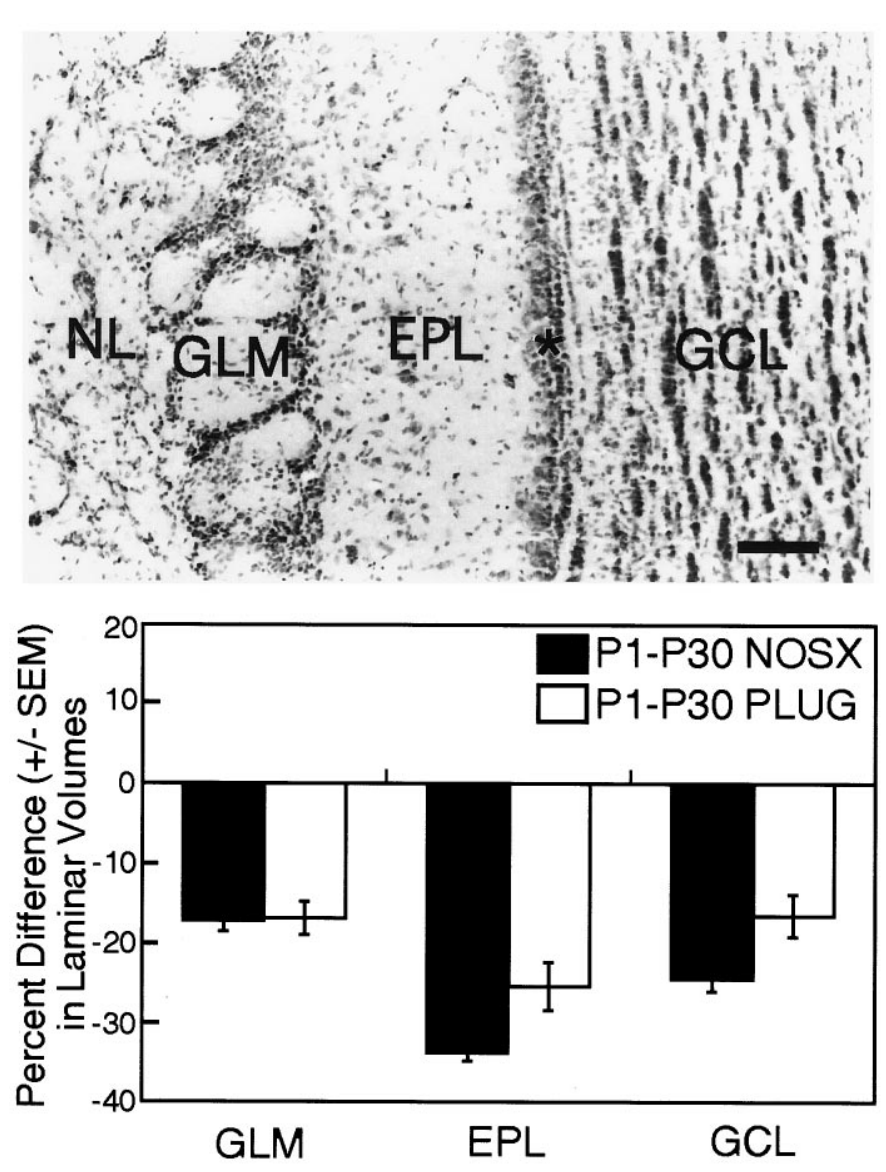

Figure 2. Photomicrograph: a Nissl-stained section depicting the layers of the olfactory bulb. Laminar volume measurements of the glomerular $(G L M)$, external plexiform $(E P L)$, and granule cell $(G C L)$ layers of experimental and control bulbs were taken and expressed as percentage differences in the graph. The mitral cell layer (asterisk) was included in the measurement of the GCL. Graph, Filled bars represent data from animals that had a naris permanently closed with cautery from P1 to P30 (Brunjes and Borror, 1983). Open bars represent animals that had a naris reversibly occluded from P1 to P30. NL, Nerve layer. Scale bar, $100 \mu \mathrm{m}$.

P1 to P30, a significant difference was observed between experimental and control bulbs for each bulb layer (GLM, EPL, and GCL $)(t=8.05,8.30$, and 6.20 respectively; $p<0.05)$. Similar size reductions were seen in animals plugged from $\mathrm{P} 1$ to $\mathrm{P} 20(t=3.58$, 5.03, and 3.65; $p<0.05$ ) (see Fig. 4).

\section{TH-ir}

Measurements of TH-ir in experimental and control bulbs provided additional evidence that reversible and permanent naris occlusion result in similar changes. $\mathrm{TH}$, the rate-limiting enzyme in the dopamine pathway, is normally expressed in juxtaglomerular cells of the olfactory bulbs, and its expression dramatically decreases after permanent unilateral naris occlusion (Baker et al., 1983). As shown in Figure 3, there was a dramatic reduction in $\mathrm{TH}$-ir in the experimental bulbs of animals reversibly occluded from P1 to P30. Optical density measurements of TH-ir in the GLM demonstrated an average $83.98 \%$ reduction in the experimental compared with the contralateral control bulb $(t=23.72$; $p<0.0001)$. Although measurements of TH-ir along the lateral aspects of the bulbs were not measured, the reduction in intensity of immunostaining did not appear as profound as that observed on the medial side. This pattern of $\mathrm{TH}$-ir is similar to that seen 
Table 2. Mean volumes (SEM) of bulb laminae $\left(\mathrm{mm}^{3}\right)$

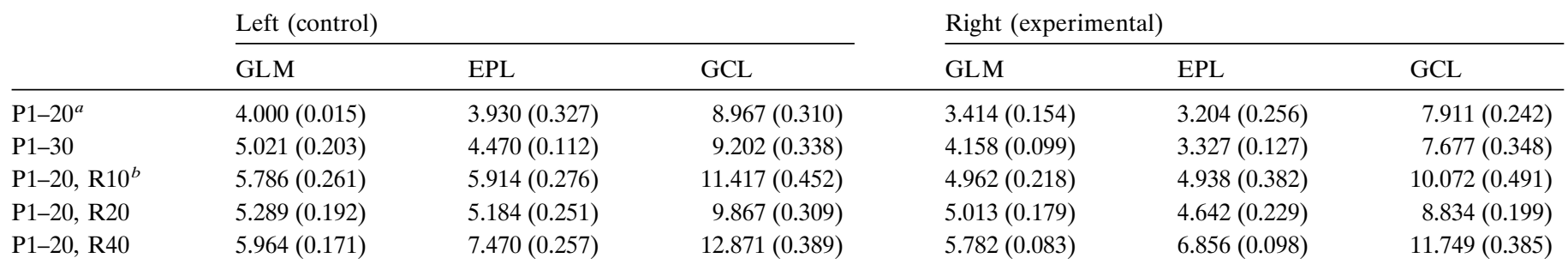

${ }^{a}$ Group fitted with plugs from P1 to P20.

${ }^{b}$ Group fitted with plugs from P1 to P20, with plug subsequently removed for $10 \mathrm{~d}$.

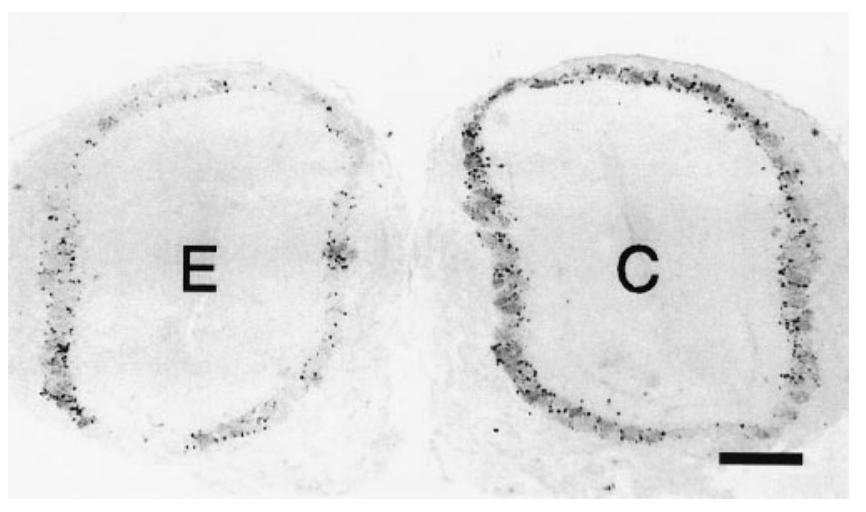

Figure 3. Photomicrograph of a coronal section through the bulbs of an animal that had a naris reversibly closed from P1 to P30 depicts immunoreactivity (ir) for tyrosine hydroxylase (TH). TH-ir is substantially reduced in the experimental bulb $(E)$ compared with the contralateral control bulb $(C)$. Scale bar, $400 \mu \mathrm{m}$.

after permanent naris closure (Stone et al., 1990; Cho et al., 1996).

\section{Olfactory mucosa}

Previous studies have shown that the thickness of the olfactory epithelium on the occluded side of the nasal cavity decreases substantially after deprivation from P1 to P20 (Farbman et al., 1988). Similar reductions in epithelial thickness were observed in animals reversibly occluded from P1 to P20 or P1 to P30 (18.14 and $17.21 \%, t=7.82$ and 9.35, respectively; $p<0.005$ ) (Fig. 4). Interestingly, mucosal measurements from rats that were occluded from P1 to P20 and recovered for $10 \mathrm{~d}$ demonstrated only a $3.70 \%$ difference in epithelial thickness between experimental and control sides, although this difference still reached statistical significance $(t=3.19 ; p<0.05)$ (Fig. 4).

\section{Recovery: bulb laminar volume measurements}

Groups of rats were reversibly occluded from P1 to P20 and then allowed access to normal olfactory stimulation for 10,20 , or $40 \mathrm{~d}$ (Fig. 5). Laminar volume measurements from rats that recovered for $10 \mathrm{~d}$ revealed that three of four animals still showed substantial reductions in all bulb lamina $(t=0.99,3.40,8.62$ for GLM, EPL, and GCL respectively; $p=0.3960$ for GLM; $p<0.05$ for EPL and GCL). In addition, recovery of bulb size was not complete after $20 \mathrm{~d}$ of recovery $(t=4.71,6.91,6.97 ; p<0.05)$. After $40 \mathrm{~d}$ of access to normal olfactory stimulation, however, volumes of the GLM, EPL, and GCL were similar in left and right bulbs of previously plugged animals $(t=1.20,0.24,1.03)$ (Fig. 5) and did not differ significantly from the sizes of these lamina in
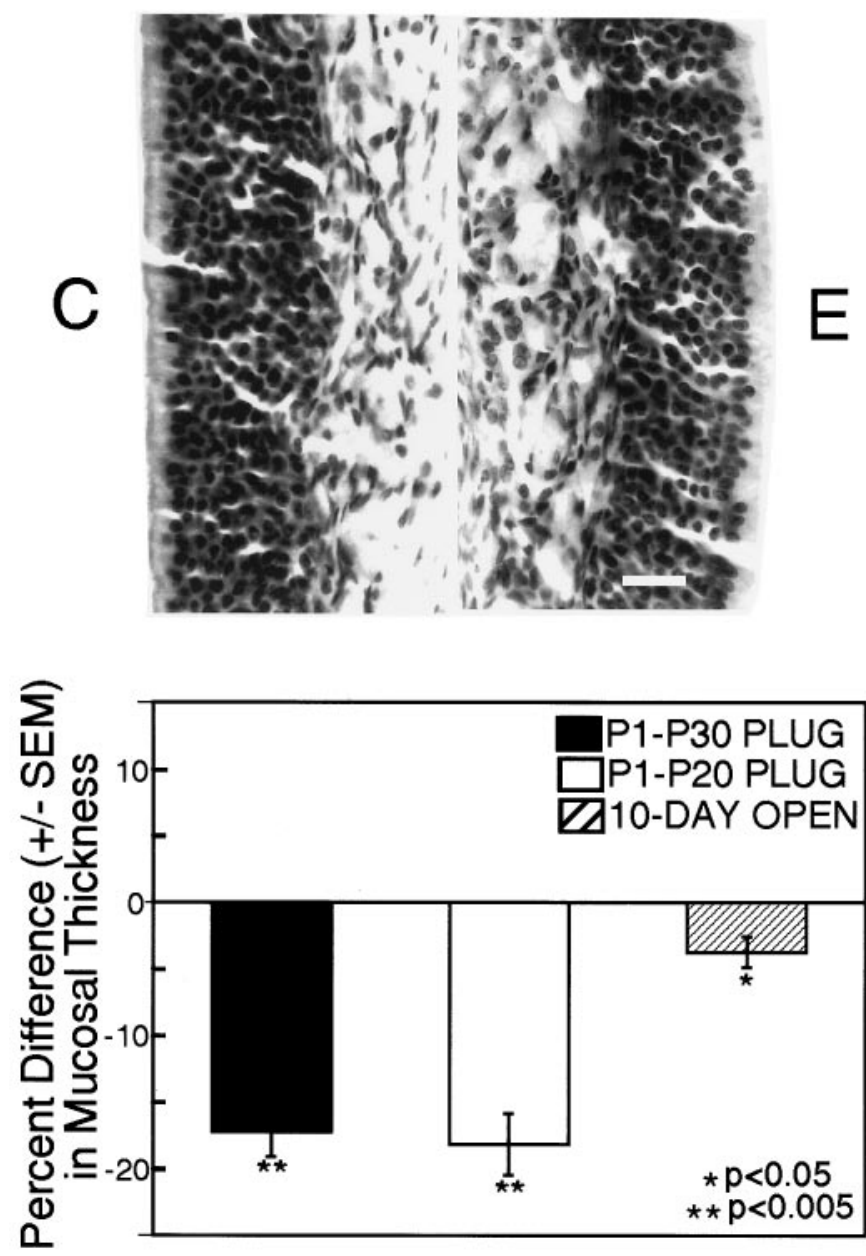

Figure 4. Top, Photomicrographs of sections through the olfactory epithelium on the experimental $(E)$ and control $(C)$ side of the septum. Sections of the mucosa were stained with hematoxylin and eosin. Bottom, Graph of percentage differences in epithelial thickness in animals that had a naris closed from P1 to P30 (filled bar), from P1 to P20 (open bar), or from P1 to P20 and then reopened for $10 \mathrm{~d}$ (hatched bar). Scale bar, $20 \mu \mathrm{m}$.

littermate control animals $(t=0.64,0.12,0.96)$, suggesting complete recovery of all bulb layers.

\section{BrdU profile counts: the fate of newly generated cells}

Animals in Group 1 (reversibly occluded from P1 to P20 with BrdU injections on P21) survived for $30 \mathrm{~d}$ before their olfactory bulbs were removed and processed for BrdU immunocytochemistry. Counts revealed an average $84.12 \%$ increase in the number 


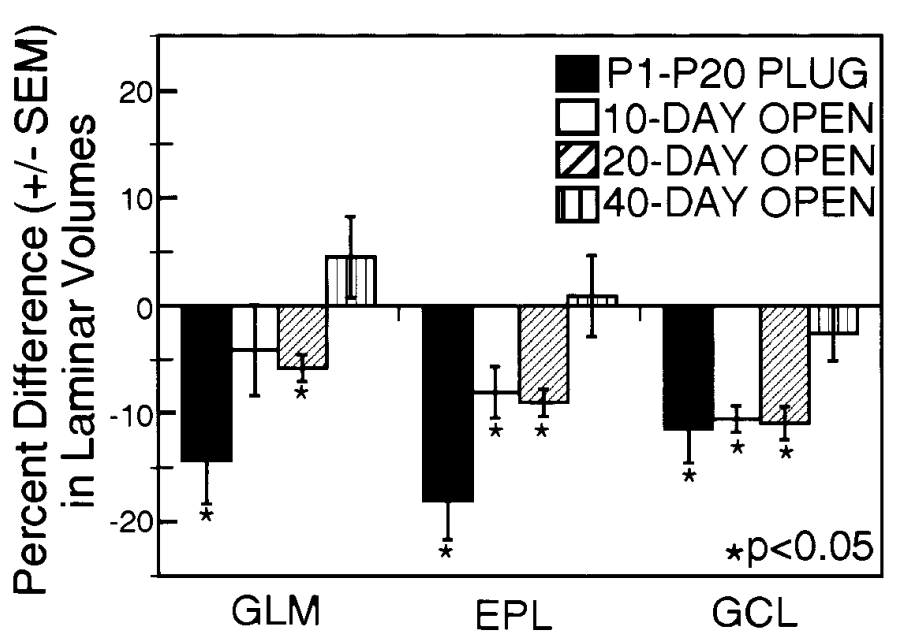

Figure 5. Graph of mean percentage differences of laminar volume measurements between experimental and control bulbs. A baseline group of animals had a naris reversibly closed from P1 to P20 (filled bars). Recovery groups had a naris closed from P1 to P20 and then opened for 10 (open bars), 20 (hatched bars), or 40 (striped bars) d.

of BrdU-ir profiles in the GLM of the previously occluded bulb compared with the contralateral control bulb $(t=12.77 ; p<$ 0.005 ) (Fig. 6A,B). Although BrdU-ir profiles in the olfactory nerve layer were not quantified because of the difficulty in determining layer boundaries, a striking increase in numbers of BrdU-ir profiles was noted in experimental compared with control bulbs (Fig. 6A,B). These profiles are probably ensheathing glia, the primary constituent of the region. Numbers of BrdU-ir profiles in the GCL did not differ significantly between previously occluded and control bulbs $(t=0.24)$ (Fig. 6, graph). Counts from the GLM and GCL of animals in Group 2 (reversibly occluded from P1 to P20, injected with BrdU twice daily from P50 to P52, and allowed to survive for $30 \mathrm{~d}$ ) revealed no differences between previously occluded and control bulbs $(t=0.95$ and 0.59 , respectively) (Fig. 6, graph).

\section{Quantification of BrdU-containing neurons in GLM of recovered bulbs}

Dual labeling with immunocytochemistry revealed that some of the BrdU-containing cells in the GLM of animals in Group 1 were immunoreactive for calretinin, a calcium-binding protein specific to neurons (Rogers, 1987) (Fig. 6C). Counts of doublelabeled cells in the GLM demonstrated a significant increase in the number of calretinin/BrdU-positive cells in the previously occluded compared with control bulbs of animals in Group $1(t=$ 8.77; $p<0.005)$.

\section{DISCUSSION}

Our data suggest that reversible naris closure with removable plugs results in similar reductions in (1) bulb volume, (2) expression of TH-ir, and (3) epithelial thickness as found after permanent naris occlusion. The most surprising aspect of this study was the reversal of the striking decrease in experimental bulb volume that occurred after normal levels of olfactory stimulation were returned. When animals were unilaterally occluded from P1 to P20 and then allowed access to normal olfactory stimulation for $40 \mathrm{~d}$, the ipsilateral bulb attained the same size as the contralateral control bulb.

As mentioned earlier, the olfactory system is unique in that it receives a constant supply of new neurons and glia that originate in the SVZ and migrate to the bulb via the rostral migratory stream (Altman, 1969; Lois and Alvarez-Buylla, 1994). To ascertain whether the number of neurons taking residence in the bulb could be modulated by changes in afferent activity, pups were unilaterally occluded with nose plugs from P1 to P20 and injected with BrdU either $24 \mathrm{hr}$ (P21) or $30 \mathrm{~d}$ (P50) after plug removal. Counts of BrdU-ir profiles in the GLM of the first group revealed a striking increase $(84.12 \%)$ in the number of newly generated cells that survived for $30 \mathrm{~d}$ in the previously occluded olfactory bulbs. Similar measures of the second, late injection group revealed no differences. The data indicated that cell addition in the GLM of the experimental bulb is temporarily enhanced in response to renewed access to normal olfactory stimulation. To verify that part of the change was attributable to incorporation of new neurons, we double-labeled tissue with immunocytochemistry for BrdU and a neuronal marker, calretinin. Counts of duallabeled cells illustrated a $27 \%$ increase in numbers of calretininpositive neurons added to the previously deprived bulb compared with controls. Therefore, the increased addition of neurons produced during the period just after plug removal may participate in the recovery of bulb size.

Although we did not detect a significant increase in numbers of cells added to the GCL of experimental bulbs at either $24 \mathrm{hr}$ or $30 \mathrm{~d}$ after naris occlusion, the substantial volume recovery that was observed strongly suggests that examinations of other time points would reveal enhanced cell addition. It is interesting to note, however, that increased cell addition was detected in the GLM, the layer that receives direct afferent input from olfactory receptor neurons. Possible reasons for this specificity are discussed below.

Recovery of epithelial thickness on the experimental side of the nasal cavity seemed to occur very soon after renewed access to normal levels of olfactory stimulation. Indeed, after only $10 \mathrm{~d}$ of normal stimulation, the reductions seen after unilateral deprivation from P1 to P20 were greatly attenuated. Although measurements of epithelial thickness provide an approximation of the relative numbers of cells in the olfactory mucosa, more detailed work is needed. In addition, an examination of epithelial cell turnover might prove interesting. Naris occlusion leads to reduced rates of cell proliferation in the olfactory receptor sheet (Farbman et al., 1988; Cummings and Brunjes, 1994). Perhaps reinstatement of access to normal olfactory stimulation rapidly changes cell proliferation within the previously deprived olfactory epithelium. A marked increase in the birth of basal cells and immature olfactory receptor neurons would explain the increase in epithelial thickness observed in this study.

\section{Comparison with other examples of postnatal neurogenesis}

For the greater part of this century, neurogenesis in the CNS was considered an exclusively prenatal and early postnatal process. Studies in the 1960s and 1970s, however, demonstrated that new neurons continue to join the olfactory bulbs and hippocampus in juvenile and adult rodents (Altman and Das, 1967; Kaplan and Hinds, 1977). The reason for this ongoing neurogenesis has been the subject of much speculation. New neurons in the dentate gyrus of the hippocampus seem to be added throughout juvenile and adult life, suggesting that they do not replace neurons that may die (Kaplan and Hinds, 1977; Bayer et al., 1982). Alternatively, work in the song-control system of birds has shown that neuronal replacement occurs in some nuclei, perhaps to play a role in song learning (Alvarez-Buylla et al., 1992). 


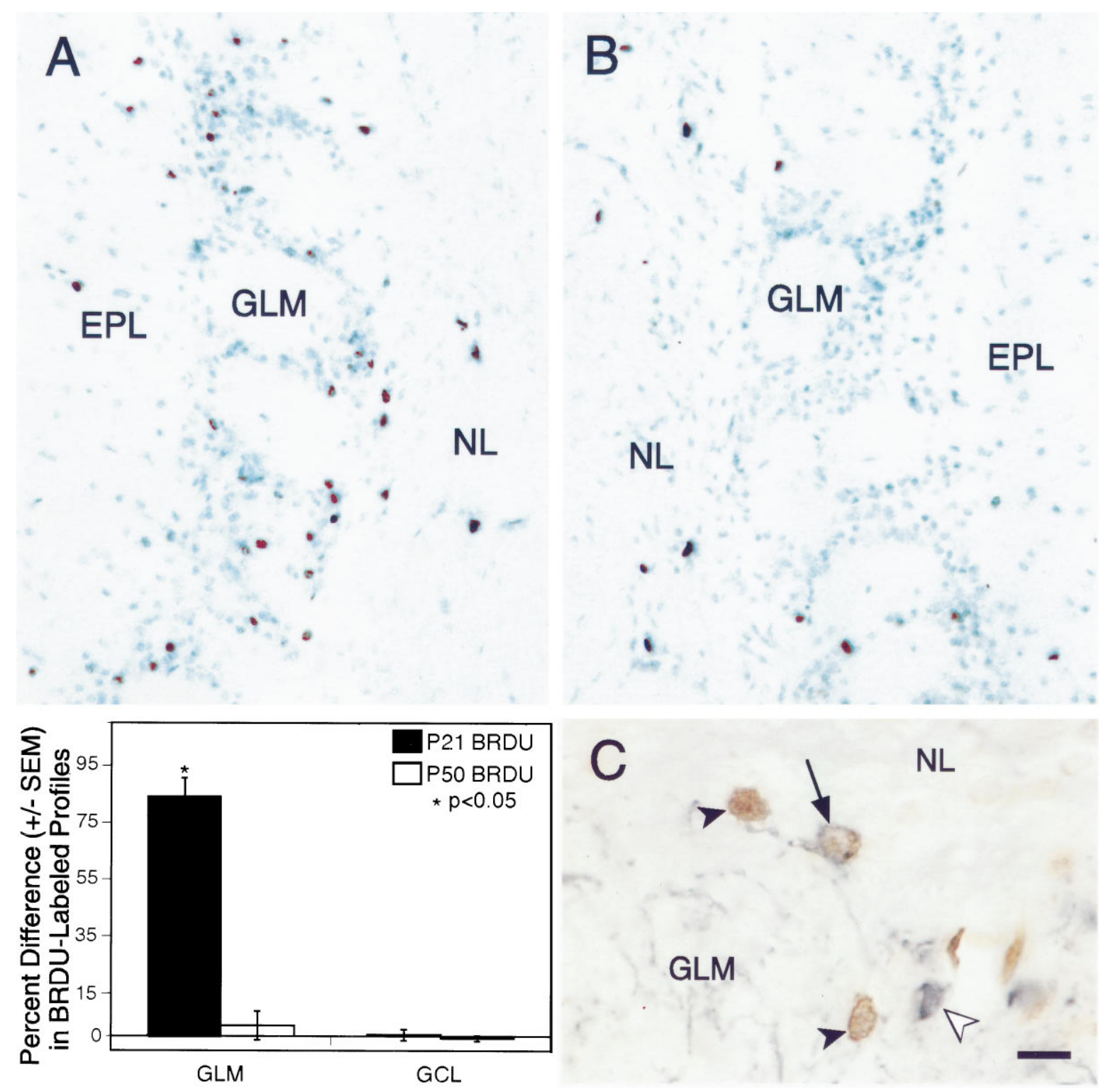

Figure 6. Photomicrographs depicting BrdU-ir along the ventromedial aspects of the bulbs of an animal that was injected with BrdU on P21, 24 hr after the naris was reopened, and survived for $30 \mathrm{~d}$. Many more BrdU-containing profiles are visible among periglomerular cells of the GLM of the experimental bulb $(A)$ compared with the contralateral control bulb $(B)$. Graph, Mean percentage differences in numbers of BrdU-containing profiles between experimental and control bulbs. Subjects had a naris closed from P1 to P20, were injected with BrdU on P21 or P50, and survived 30 d after the BrdU injections. $G L M$, Glomerular layer; $G C L$, granule cell layer. $C$, Photomicrograph of a section adjacent to the one shown in $B$ that was processed through immunocytochemistry for BrdU (brown) and calretinin (blue). A double-labeled cell (arrow) is visible along with single-labeled cells that were immunoreactive for either BrdU ( filled arrowheads) or calretinin (open arrowhead). A process from the double-labeled cell can be seen extending in the direction of a single-labeled BrdU-ir cell. GLM, Glomerular layer; $N L$, nerve layer. Scale bar (shown in $C$ ): $A, B, 40 \mu \mathrm{m} ; C, 10 \mu \mathrm{m}$.

The function of continued neuronal addition to the olfactory bulbs is unknown. One possibility is that new interneurons are simply added to the bulbs, as they are in the hippocampus. Yet, although increases in numbers of interneurons have been reported in adult rats (Hinds and McNelly, 1977, 1981; Kaplan et al., 1985), a substantial amount of granule cell death has also been observed, suggesting that newly generated neurons may replace dying ones (Kaplan et al., 1985). If neuronal replacement does occur in the juvenile and adult olfactory bulb, then understanding the regulation of this process is of utmost importance and could have implications for facilitating neuronal replacement in other brain regions.

Conflicting results exist as to whether the proliferation of neu- roblasts destined for the bulb can be influenced by exogenous factors such as afferent activity levels. Permanent naris closure does not reduce proliferation levels in early postnatal rats (Frazier-Cierpial and Brunjes, 1989); however, the manipulation may decrease proliferation in adult mice (Corotto et al., 1994). Perhaps the regulation of SVZ cell proliferation changes from a preprogrammed state during bulb growth to an activitydependent state during bulb maintenance. Regardless of whether proliferation can be regulated, substantial evidence from many brain regions indicates that activity is important for cell survival.

Previous results suggest that neuronal survival in developing and adult rodents depends on afferent activity (Frazier-Cierpial and Brunjes, 1989; Henegar and Maruniak, 1991; Woo and Leon, 
1991). In developing rats, for example, naris closure leads to decreased neuronal survival (Frazier-Cierpial and Brunjes, 1989; Najbauer and Leon, 1995). Odor learning, however, leads to greater numbers of cells in particular foci within the glomerular layer (Woo and Leon, 1991). These areas are associated with increased activity, as measured by enhanced ${ }^{14} \mathrm{C}$-2-deoxyglucose (2-DG) uptake (Woo et al., 1987; Woo and Leon, 1991). Although the increase may have resulted from neuronal rearrangement, a more intriguing possibility is that activity in these foci led to enhanced cell survival. The current study suggests that the probability of survival for newly formed neurons may be enhanced when normal levels of stimulation from the environment are reinstated after deprivation. Because this phenomenon follows a period of neuronal loss and includes the addition of new neurons to the experimental olfactory bulb, we suggest that this change might signify neuronal replacement within the juvenile CNS.

\section{How might increased cell addition occur?}

Renewed access to environmental stimulation results in a substantial increase in odor-induced activity. Surgical opening of nares that were closed from P2 to P22 in rats leads to enhanced uptake of 2-DG and increased responsiveness in mitral/tufted $(\mathrm{M} / \mathrm{T})$ cells after stimulation with an odor (Guthrie et al., 1990). These findings suggest that bulbs that have just gone from a deprived to a nondeprived state are super-sensitive to olfactory experiences. How might this condition translate into increased cellular addition?

Several factors may be involved. One possibility is that on reopening the closed naris, activity-induced branching occurs among axonal terminals of olfactory receptor neurons (ORNs) and dendrites of $\mathrm{M} / \mathrm{T}$ cells. The elaboration of ORN axon terminals might stimulate an increase in the number of ensheathing cells in olfactory nerve layer. The increase in presynaptic terminals might also enhance dendrogenesis in $\mathrm{M} / \mathrm{T}$ and juxtaglomerular cells, ultimately increasing the available synaptic space.

Ultrastructural changes and synapse formation can be influenced by activity levels in other brain regions. For example, rapid increases in structural and synaptic formations have been observed after kindling in vivo and long term potentiation (LTP) in vitro in the adult rat hippocampus (Lee et al., 1980; Chang and Greenough, 1984). LTP induction in the hippocampus is dependent on the activation of NMDA-type glutamate receptors and has often been associated with learning and memory. Anatomical and physiological data suggest that glutamate is a transmitter used by ORNs and that both NMDA- and non-NMDA-type glutamate receptors are present on $\mathrm{M} / \mathrm{T}$ dendrites (Brennan et al., 1990; Sassoè-Pognetto et al., 1993; Berkowicz et al., 1994). Studies of olfactory learning in developing rats suggest that blocking NMDA-type glutamate receptors reduces the neurobehavioral response to olfactory preference training (Lincoln et al., 1988). Therefore, activity-dependent mechanisms involving NMDAtype glutamate receptors may underlie the changes that result in increased cell addition in previously deprived bulbs.

\section{Conclusions}

The present findings suggest that the deprived olfactory bulb is able to regenerate after the restoration of normal environmental stimulation. The availability of undifferentiated neuroblasts that travel from the SVZ to the olfactory bulbs throughout life provides the bulb with increased opportunities for change. The constant neuronal turnover of sensory neurons within the olfactory epithelium may also be related to the ongoing addition of new cells to the olfactory bulb. Thus, the bulb may serve as a model for investigating potential mechanisms of neuronal replacement in the mammalian CNS.

\section{REFERENCES}

Altman J (1963) Autoradiographic investigation of cell proliferation in the brains of rats and cats. Anat Rec 145:573-591.

Altman J (1969) Autoradiographic and histological studies of postnatal neurogenesis. IV. Cell proliferation and migration in the anterior forebrain, with special reference to persisting neurogenesis in the olfactory bulb. J Comp Neurol 137:433-458.

Altman J, Das GD (1967) Postnatal neurogenesis in the guinea-pig. Nature 214:1098-1101.

Alvarez-Buylla A, Ling C-Y, Nottebohm F (1992) High vocal center growth and its relation to neurogenesis, neuronal replacement and song acquisition in juvenile canaries. J Neurobiol 23:396-406.

Baker H, Kawano T, Margolis FL, Joh TH (1983) Transneuronal regulation of tyrosine hydroxylase expression in olfactory bulb of mouse and rat. J Neurosci 3:69-78.

Bayer SA (1983) 3H-thymidine-radiographic studies of neurogenesis in the rat olfactory bulb. Exp Brain Res 50:329-340.

Bayer SA, Yackel JW, Puri PS (1982) Neurons in the rat dentate gyrus granular layer substantially increase during juvenile and adult life. Science 216:890-892.

Berkowicz DA, Trombley PQ, Shepherd GM (1994) Evidence for glutamate as the olfactory receptor cell neurotransmitter. J Neurophysiol $71: 2557-2561$.

Brennan P, Kaba H, Keverne EB (1990) Olfactory recognition: a simple memory system. Science 250:1223-1226.

Brunjes PC (1994) Unilateral naris closure and olfactory system development. Brain Res Rev 19:146-160.

Brunjes PC, Borror MJ (1983) Unilateral odor deprivation: differential effects due to time of treatment. Brain Res Bull 11:501-503.

Brunjes PC, Jazaeri A, Sutherland MJ (1992) Olfactory bulb organization and development in Monodelphis domestica (grey short-tailed opossum). J Comp Neurol 320:544-554.

Chang F-LF, Greenough WT (1984) Transient and enduring morphological correlates of synaptic activity and efficacy change in the rat hippocampal slice. Brain Res 309:35-46.

Cho JY, Min N, Franzen L, Baker H (1996) Rapid down-regulation of tyrosine hydroxylase expression in the olfactory bulb of naris-occluded adult rats. J Comp Neurol 369:264-276.

Corotto FS, Henegar JR, Maruniak JA (1994) Odor deprivation leads to reduced neurogenesis and reduced neuronal survival in the olfactory bulb of the adult mouse. Neuroscience 61:739-744.

Cummings DM, Brunjes PC (1994) Changes in cell proliferation in the developing olfactory epithelium following neonatal unilateral naris occlusion. Exp Neurol 128:124-128.

Farbman AI (1992) Cell biology of olfaction. Cambridge: Cambridge UP.

Farbman AI, Brunjes PC, Rentfro L, Michas J, Ritz S (1988) The effect of unilateral naris occlusion on cell dynamics in the developing rat olfactory epithelium. J Neurosci 8:3290-3295.

Frazier-Cierpial LL, Brunjes PC (1989) Early postnatal cellular proliferation and survival in the olfactory bulb and rostral migratory stream of normal and unilaterally odor-deprived rats. J Comp Neurol 289:481-492.

Gratzner HG (1982) Monoclonal antibody to 5-bromo- and 5-iododeoxyuridine: a new reagent for detection of DNA replication. Science 218:474-475.

Guthrie KM, Wilson DA, Leon M (1990) Early unilateral deprivation modifies olfactory bulb function. J Neurosci 10:3402-3412.

Henegar JR, Maruniak JA (1991) Quantification of the effects of longterm unilateral naris closure on the olfactory bulbs of adult mice. Brain Res 568:230-234.

Hinds JW, McNelly MA (1977) Aging of the rat olfactory bulb: growth and atrophy of constituent layers and changes in size and number of mitral cells. J Comp Neurol 171:345-368.

Hinds JW, McNelly MA (1981) Aging in the rat olfactory system: correlation of changes in the olfactory epithelium and olfactory bulb. J Comp Neurol 203:441-453. 
Kaplan MS, Hinds JW (1977) Neurogenesis in the adult rat: electron microscopic analysis of light radioautographs. Science 197:1092-1094.

Kaplan MS, McNelly MA, Hinds JW (1985) Population dynamics of adult-formed granule neurons of the rat olfactory bulb. J Comp Neurol 239:117-125.

Kucharski D, Hall WG (1987) New routes to early memories. Science 238:786-788.

Lee KS, Schottler F, Oliver M, Lynch G (1980) Brief bursts of highfrequency stimulation produce two types of structural change in rat hippocampus. J Neurophysiol 44:247-258.

Lincoln J, Coopersmith R, Harris EW, Cotman CW, Leon M (1988) NMDA receptor activation and early olfactory learning. Dev Brain Res 39:309-312.

Lois C, Alvarez-Buylla A (1994) Long-distance neuronal migration in the adult mammalian brain. Science 264:1145-1148.

Luskin MB (1993) Restricted proliferation and migration of postnatally generated neurons derived from the forebrain subventricular zone. Neuron 11:173-189.

Meisami E (1976) Effects of olfactory deprivation on postnatal growth of the rat olfactory bulb utilizing a new method for production of neonatal anosmia. Brain Res 107:437-444.
Najbauer J, Leon M (1995) Olfactory experience modulates apoptosis in the developing olfactory bulb. Brain Res 674:245-251.

Rogers JH (1987) Calretinin: a gene for a novel calcium-binding protein expressed principally in neurons. J Cell Biol 105:1343-1353.

Sassoè-Pognetto M, Cantino D, Panzanelli P, di Cantogno LV, Giustetto M, Margolis FL, De Biasi S, Fasolo A (1993) Presynaptic colocalization of carnosine and glutamate in olfactory neurones. NeuroReport 5:7-10.

Stone DM, Wessel T, Joh TH, Baker H (1990) Decrease in tyrosine hydroxylase, but not aromatic L-amino acid decarboxylase, messenger RNA in rat olfactory bulb following neonatal, unilateral odor deprivation. Mol Brain Res 8:291-300.

Widner H (1993) Immature neural tissue grafts in Parkinson's disease. Acta Neurol Scand [Suppl] 146:43-45.

Woo CC, Leon M (1991) Increase in focal population of juxtaglomerular cells in the olfactory bulb associated with early learning. J Comp Neurol 305:49-56.

Woo CC, Coopersmith R, Leon M (1987) Localized changes in olfactory bulb morphology associated with early olfactory learning. J Comp Neurol 263:113-125. 